\title{
EDITOR'S ACKNOWLEDGEMENTS
}

The Editor gratefully acknowledges the help of her co-editor (Prof. Cathy Morgan), various members of the Publications Board, all the referees, the copy-editor (Leofranc HolfordStrevens) and the typesetter (Mark Heslington) in the preparation of this volume. Mr Petros Kounouklas and Dr. Y. Galanakis kindly provided Greek translations for some of the abstracts; Ms Elaine Massung helped in many practical ways. 